\title{
Development and Characterization of SSR Markers in Proso Millet Based on Switchgrass Genomics
}

\author{
Santosh G. Rajput, Tammy Plyler-Harveson, Dipak K. Santra* \\ Panhandle Research and Extension Center, University of Nebraska-Lincoln, Scottsbluff, USA. \\ Email: *dsantra2@unl.edu
}

Received November $24^{\text {th }}, 2013$; revised December $25^{\text {th }}, 2013$; accepted January $11^{\text {th }}, 2014$

Copyright (c 2014 Santosh G. Rajput et al. This is an open access article distributed under the Creative Commons Attribution License, which permits unrestricted use, distribution, and reproduction in any medium, provided the original work is properly cited. In accordance of the Creative Commons Attribution License all Copyrights (C) 2014 are reserved for SCIRP and the owner of the intellectual property Santosh G. Rajput et al. All Copyright (C) 2014 are guarded by law and by SCIRP as a guardian.

\begin{abstract}
Proso millet (Panicum miliaceum) has high water use efficiency (WUE), a short growing-season, and is highly adapted to a semi-arid climate. Genomic resources for proso millet are very limited. Large numbers of DNA markers and other genomic tools in proso millet can readily be developed by using genomic resources in related grasses. The objectives of the present report were to 1) test and characterize switchgrass SSR markers for use in proso millet, and 2) elucidate repeat-motifs in proso millet based on new SSR marker analysis. A total of 548 SSR markers were tested on 8 proso millet genotypes. Out of these, 339 amplified SSR markers in proso millet. This showed that $62 \%$ of the switchgrass SSR markers were transferable to proso millet. Of these 339 markers, 254 were highly polymorphic among the 8 proso genotypes. The resolving power of these 254 polymorphic SSR markers ranged from 0.25 - 14.75 with an average of 2.71 . The 254 polymorphic SSR markers amplified 984 alleles in the ranges of $50 \mathrm{bp}$ to $1300 \mathrm{bp}$. The majority of the SSR markers (221 of 254) amplified dinucleotide repeats. Based on SSR marker analysis, AG/GA was the most abundant repeat-motifs in proso millet. Switchgrass genomic information seems to be the most useful for developing DNA markers in proso millet. Markers developed in this study will be helpful for linkage map construction, mapping agronomic traits and future molecular breeding efforts in proso millet.
\end{abstract}

\section{KEYWORDS}

Millet; Minor Crops; Comparative Genomics; Molecular Breeding

\section{Introduction}

Proso millet (Panicum miliaceum L.) is reported to have been domesticated about 10,000 years ago in central and Eastern Asia and made its way from China to the Black Sea region of Europe by 5000 BC [1]. German-Russian immigrants introduced proso millet to North America in 1875 [2]. Proso millet is known as common millet, millet, and hog millet in the United States of America (USA); broomcorn millet in China; common millet in Japan, Korea, and other Pacific Asian countries; "hersey" millet in Germany; and French white in France [3]. It is currently grown in Asia, Australia, North America, Europe, and Africa [3-5].

Proso millet is the most suitable rotational crop in ma-

*Corresponding author. jority of dryland wheat producing areas in semi-arid High Plains of the USA [6]. It is grown mostly in Colorado, Nebraska, and South Dakota and to a lesser extent in North Dakota, Montana, Wyoming, Kansas, Oklahoma, Texas, Minnesota, Wisconsin, Iowa, Michigan, North Carolina, Georgia, and Florida [7]. Proso millet is highly diversified, has excellent nutritional properties and could become an important crop for food diversification [8]. It is used primarily for human consumption in Asia. In the USA, it is grown primarily for birdseed and livestock $[8,9]$.

A very limited number of DNA markers are reported in proso millet. A few RAPD and AFLP markers were reported in proso millet for genetic diversity analysis [10-12]. Among all PCR-based markers, microsatellites or simple sequence repeat (SSR) markers are the most 
informative and useful because they are co-dominant, multi-allelic, highly reproducible, abundant and are evenly distributed in the plant genome [13,14]. Hu et al. (2009) [15] identified 46 SSRs markers from rice, wheat, oat and barley for genetic diversity analysis in proso millet. Cho et al. (2010) [16] developed 25 SSR markers from proso millet BAC library. To date only about 100 SSR markers are available, which is inadequate for proso millet genetic and breeding research. But unfortunately, developing SSR markers is expensive and a time-consuming task for any crop $[17,18]$. Comparative genomics has great potential to speed up development of genomic tools in minor crops such as proso millet using genome resources of other major crops such as rice, maize, sorghum and foxtail millet [19]. SSR markers developed in related species were successfully used in sorghum, and bermudagrass $[20,21]$.

Switchgrass, where enormous genomic resource is available, is taxonomically the closest species to proso millet [22]. A detailed genome map consisting of more than 2000 SSR markers is available in switchgrass [2325]. Therefore, switchgrass genomic data are the most suitable for developing DNA markers for proso millet in a rapid and inexpensive way. The objectives of this report were to 1 ) test and characterize switchgrass SSR markers for using in proso millet, and 2) elucidate repeat-motifs in proso millet based on the new SSR marker analysis.

\section{Materials and Methods}

\subsection{Plant Materials and SSR Markers}

A set of 8 proso millet genotypes were used for testing efficacy of the switchgrass SSR markers. Plants were grown in 4-inch pots filled with Sunshine Mix 2 Basic Professional Growing Mix (Westco, Morill, NE, USA) in a greenhouse at the University of Nebraska, Panhandle Research and Extension Center, Scottsbluff. Leaf tissues of 21-day-old plants were used for DNA extraction.

A total of 548 SSR markers ( 540 genomic \& 8 EST) of switchgrass were used $[23,25]$ These SSR markers are evenly distributed throughout the switchgrass genome. Primers of these SSR markers were synthesized from MWG Biotech (Huntsville, AL) based on sequence reported by Wang et al. 2011 [25]. These 548 SSR markers were tested on 8 proso millet genotypes (Earlybird, Huntsman, Panhandle, Horizon, Plateau, PI436625, PI436626 and PI 463473) to determine potential polymorphic markers. Polymorphic SSR motif repeats were analyzed based on the data published by Wang et al. 2011 [25] and Okada et al. 2010 [24].

\subsection{DNA Extraction and SSR Marker Analysis}

Genomic DNA was isolated by micro prep CTAB DNA isolation method using about $100 \mathrm{mg}$ leaf tissues [26].
DNA quality and quantity were checked on a $0.8 \%$ agarose gel using 0.5XTBE buffer and visualized under UV transillumination. Concentration of DNA in each sample was determined comparing intensity of bands of Lambda/ HindIII DNA markers. PCR consisted of 10 ulreaction volume containing $1 \times$ reaction buffer, $1 \mathrm{U}$ Taq polymerase, 200 uMdNTPs, $2 \mathrm{mM} \mathrm{MgCl}_{2}, 5$ pmoles primers, 20 ng DNA. PCR cycle was: initial denaturation of $5 \mathrm{~min}$ at $94^{\circ} \mathrm{C}$; followed by 39 cycles of $45 \mathrm{~s}$ at $94^{\circ} \mathrm{C}, 50 \mathrm{~s}$ at $55^{\circ} \mathrm{C}$, and $45 \mathrm{~s}$ at $72^{\circ} \mathrm{C}$ and a final $10 \mathrm{~min}$ extension at $72^{\circ} \mathrm{C}$. PCR was done using BioRad S1000 Thermal cycler. PCR amplified products were separated in a $2 \%$ agarose gel using 0.5XTBE buffer and visualized under UV transillumination after staining with ethidium bromide @0.5 ug/ml. Markers (>50 bp) were scored manually using gel image and band size was determined considering 25 bp size difference between the bands. A SSR marker was considered polymorphic if two DNA bands of different sizes were observed at least in two genotypes. DNA bands of different sizes were considered different alleles and DNA bands of same size were considered as the same allele. Resolving power (Rp) of each SSR marker was calculated by following formula $\mathrm{Rp}=\sum I_{b}$ and $I_{b}=1$ $-[2 \times(0.5-p)]$ where $I_{b}$ is the allele informativeness and $p$ is the proportion of accessions sharing the $I$ allele [27]. The NCBI nucleotide database was searched for homology analysis with nucleotide sequences in other crops using primers sequences of the polymorphic SSR markers.

\section{Results and Discussion}

\subsection{Switchgrass SSRs in Proso Millet}

Of the 548 switchgrass SSR markers screened, 339 (62\%) amplified and 209 markers (38\%) did not amplify in proso millet. Of the 339 amplified markers, 254 (46\%) were polymorphic. Many of these 254 markers were found to be highly polymorphic among the set of 8 proso millet genotypes as represented by one such SSR marker PVCA 21-22 (Figure 1). The high polymorphic nature of these markers was observed also from their high resolving power of each SSR marker, which ranged from 0.25 14.75 with an average 2.71 (Figure 2). A smallest resolving power of 0.25 was shown by six markers (PVCA 1431-32, PVCA 299-300, PVCA 985-86, PVCA 151-52, PVCA 415-16 and NFSG-246) while the highest resolving power of 14.75 was observed for PVCA 1387-88. As reported by Prevost and Wilkinson (1999) [27], higher resolving power is directly linked to the informativeness of the marker. Based on our data it seems that our SSR markers have moderate resolving power which can be useful to select the efficient marker set for future studies. The names of the 254 polymorphic SSR markers and their characteristics (allele sizes and Resolving pow- 
er (Rp) values) are given in Table 1 . We consider that the 339 SSR markers covered most of the proso millet genome since these markers were selected from most of the chromosomal regions of switchgrass. Lack of amplification of the proso millet genome by $38 \%$ of 548 switchgrass SSR markers could be due to insertion and/ or deletion present in SSR motifs, or point mutations and/or deletions in flanking repeats [28].

Approximately $98 \%$ of the polymorphic markers were genomic SSR markers and only $2 \%$ were EST SSR markers. This is similar to other reports in proso millet and in switchgrass $[16,25]$. High percentages of transferability of switchgrass markers into proso millet supports the utilization of cross species SSR markers as a useful resource for developing markers in minor crops where very limited or no work has been done at the molecular level. Similar results were obtained in other crops such as sorghum, bermudagrass, napiergrass, finger millet and other grasses $[20,21,29,30]$. Such high level of transferability is probably because many genomic regions are

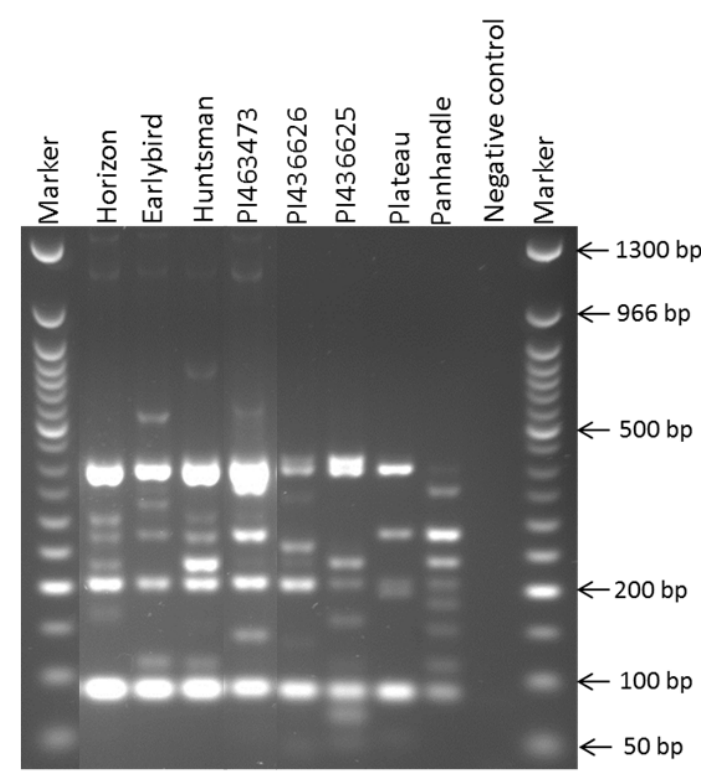

Figure 1. Polymorphic profile of switch grass SSR marker PVCA 21-22 in 8 proso genotypes $(M=50$ bp NEB DNA Ladder, NC = Negative control).

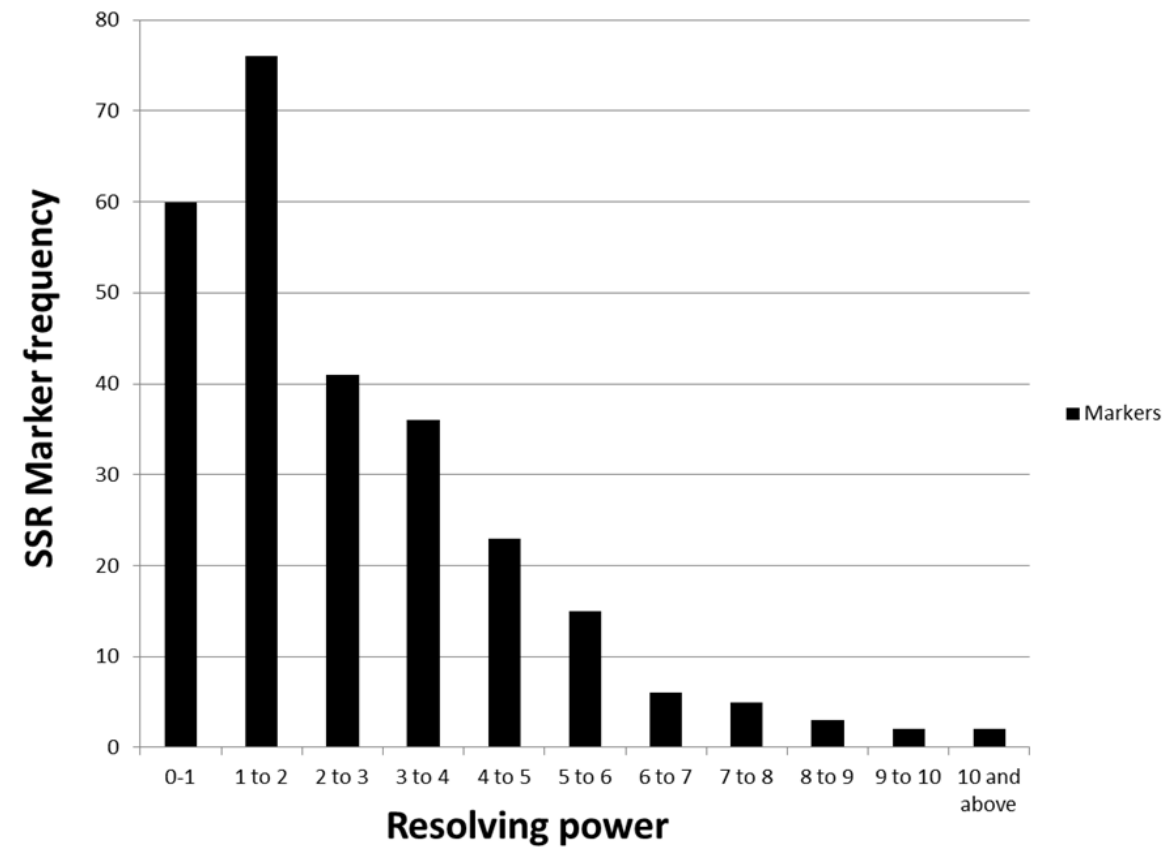

Figure 2. Resolving power values of 254 polymorphic SSR markers in proso millet. 
Table 1. Characteristics of switchgrass SSR markers, which were polymorphic in proso millet.

\begin{tabular}{|c|c|c|c|}
\hline Sr. no. & Marker Name $^{\#}$ & Observed allele size in proso millet (bp) & $\begin{array}{l}\text { Resolving power } \\
\text { (Rp) }\end{array}$ \\
\hline 1 & PVCA1763-64 & 400,350 & 0.75 \\
\hline 2 & PVCA1761-62 & $600,500,350$ & 0.75 \\
\hline 3 & PVCA1751-52 & $400,350,300$ & 1 \\
\hline 4 & PVCA1737-38 & 600,200 & 0.5 \\
\hline 5 & PVCA1717-18 & $1000,500,250$ & 0.75 \\
\hline 6 & PVCA1525-26 & $325,250,175$ & 4.5 \\
\hline 7 & PVCA1513-14 & $650,250,200$ & 2.5 \\
\hline 8 & PVCA1431-32 & 650 & 0.25 \\
\hline 9 & PVCA1429-30 & 75,50 & 1 \\
\hline 10 & PVCA1263-64 & $500,400,250,75$ & 1.75 \\
\hline 11 & PVCA1217-18 & 150 & 1 \\
\hline 12 & PVCA1181-82 & 600,150 & 1.25 \\
\hline 13 & PVCA993-94 & 1300,250 & 1 \\
\hline 14 & PVCA989-90 & $1000,800,550,525,325,225,150,75$ & 3.5 \\
\hline 15 & PVCA751-52 & $900,750,200,100$ & 4 \\
\hline 16 & PVCA733-34 & $750, \quad 275$ & 2 \\
\hline 17 & PVCA445-46 & 600,300 & 1.25 \\
\hline 18 & PVCA407-08 & $450, \quad 250, \quad 200$ & 2.5 \\
\hline 19 & PVCA401-402 & $1300,400, \quad 350, \quad 250, \quad 200$ & 6 \\
\hline 20 & PVCA1647-48 & $1200, \quad 400,325, \quad 250,125$ & 3.75 \\
\hline 21 & PVCA215-16 & $525,500,75,50$ & 3.5 \\
\hline 22 & PVCA201-02 & $750,700,350,225,200,175,100$ & 7.75 \\
\hline 23 & PVCA155-56 & $175,150,100$ & 4.5 \\
\hline 24 & PVCA137-38 & $450,300,335,175,150$ & 3 \\
\hline 25 & PVCA255-56 & 1000, 700, 600, 350, 200, 100 & 4 \\
\hline 26 & PVCA343-44 & 1300, 500, 250, 200 & 4.25 \\
\hline 27 & PVCA1535-36* & $375,350,300,150,75,50$ & 6.25 \\
\hline 28 & PVCA1119-20 & $300,275,225,150$ & 4.5 \\
\hline 29 & PVCA1107-08 & 1000,150 & 0.5 \\
\hline 30 & PVCA1091-92 & 300,75 & 2 \\
\hline 31 & PVCA47-48 & $1300,450,400$ & 1 \\
\hline 32 & PVCA1799-1800 & $700,600,425,400,325,253,175,125,75$ & 6.5 \\
\hline 33 & PVCA1659-60 & 250 & 1 \\
\hline 34 & PVCA1701-02 & $900,400,300,250,225,200,175,75$ & 3.75 \\
\hline 35 & PVCA1687-88 & $1000,800,650,425,200,100,75,50$ & 6 \\
\hline 36 & PVCA1257-58 & 1300, 1200, 650, 450, 400, 325, 225 & 7.25 \\
\hline 37 & PVCA1679-80 & 1000, 750, 600, 400, 250, 200 & 4.75 \\
\hline 38 & PVCA21-22 & $700,600,500,400,350,150$ & 4.25 \\
\hline 39 & PVCA57-58 & $475,300,175,150,100$ & 4.5 \\
\hline 40 & PVCA167-68 & $1300,1200,350,250,200,150,75$ & 7.5 \\
\hline 41 & PVCA285-86 & $750,550,450,400,300,275,200,175,150,100$ & 7.5 \\
\hline 42 & PVCA299-300 & 125 & 0.25 \\
\hline 43 & PVCA429-30 & $700,500,150,125,100,75,50$ & 4 \\
\hline 44 & PVCA1259-60 & $800,750,700,600,550,350,150,100,75$ & 4.5 \\
\hline 45 & PVCA639-40 & $900,700,450,425,325,300,250,175,150,100$ & 6.5 \\
\hline 46 & PVCA687-88 & $800,750,700,650,475,450,400,300,175,125$ & 5.25 \\
\hline 47 & PVCA183-84 & $1200,1000,750,650,375,350,200,150,100,50$ & 4.25 \\
\hline 48 & PVCA191-92 & $1300,1200,1000,700,400,300,250,225,175,100$ & 9 \\
\hline
\end{tabular}




\section{Continued}

\begin{tabular}{|c|c|c|c|}
\hline 49 & PVCA199-200 & $750,700,450,400,250,225,175,125,100,75$ & 8.25 \\
\hline 50 & PVCA951-52 & $1000,650,600,550,375,350,300,275,250,100,75,50$ & 4.75 \\
\hline 51 & PVCA1055-56 & $900,400,350,300,250,200,150$ & 5.25 \\
\hline 52 & PVCA1105-06 & $650,600,500,400,275,250,175,150$ & 3 \\
\hline 53 & PVCA1221-22 & $1000,750,700,400,350,300,275,250,225,200,175,150,125,100,75,50$ & 8.75 \\
\hline 54 & PVCA1267-68 & $950,600,125,100$ & 1.75 \\
\hline 55 & PVCA1279-80 & $900,550,500,350,325,250,150$ & 7 \\
\hline 56 & PVCA1387-88 & $1300,650,500,375,350,300,225,175,150,125,100,75,50$ & 14.75 \\
\hline 57 & PVCA1421-22 & $500,250,125,100,75$ & 1.5 \\
\hline 58 & PVCA1449-50 & $275,250,225,200,50$ & 2.5 \\
\hline 59 & PVCA1479-80 & $1300,900,550,300,200,175,75,50$ & 3 \\
\hline 60 & PVCA1555-56 & $650,600,400,375,225,200,150,100$ & 9.25 \\
\hline 61 & PVCA1565-66 & $650,600,350,325,300,275,250,225,150,100$ & 9.25 \\
\hline 62 & PVCA1571-72 & $1100,900,850,800,750,650,625,550,400,350,325,175,150,125,75$ & 7.25 \\
\hline 63 & PVCA1573-74 & $500,400,325,300,250,200,175,150,100$ & 6 \\
\hline 64 & PVCA1651-52 & $900,700,525,450,400,325,300,275,250,100,75,50$ & 5.5 \\
\hline 65 & PVCA1731-32* & $750,600,500,425,400,350,300,325,300,225,175,150,125,100,75,50$ & 10.5 \\
\hline 66 & PVCA1-2 & 700,250 & 0.75 \\
\hline 67 & PVCA109-10 & 700, 325, 300, 200 & 1.5 \\
\hline 68 & PVCA161-62 & $500,400,150$ & 1.75 \\
\hline 69 & PVCA231-32 & $700,500,300,150$ & 2.75 \\
\hline 70 & PVCA259-60 & $600,500,300,150$ & 3.25 \\
\hline 71 & PVCA271-72 & $500,325,250,100$ & 1.25 \\
\hline 72 & PVCA275-76 & 1300, 750, 300, 200, 150, 100 & 4.25 \\
\hline 73 & PVCA297-98 & $500,350,300,250$ & 1.25 \\
\hline 74 & PVCA301-02 & 750, 250, 200 & 3 \\
\hline 75 & PVCA345-46 & 700,350 & 0.5 \\
\hline 76 & PVCA347-48 & $325,200,150$ & 1 \\
\hline 77 & PVCA445-46 & $175,125,100$ & 1.5 \\
\hline 78 & PVCA495-96 & $800,300,150$ & 1 \\
\hline 79 & PVCA533-34 & $700,500,400$ & 0.75 \\
\hline 80 & PVCA541-42 & $750,700,350,200,150$ & 2.75 \\
\hline 81 & PVCA557-58 & $550,500,300$ & 1.25 \\
\hline 82 & PVCA571-72 & 400,300 & 0.75 \\
\hline 83 & PVCA579-80 & $700,650,600,500,200$ & 2.75 \\
\hline 84 & PVCA609-10 & $750,650,450,350,150$ & 4.75 \\
\hline 85 & PVCA615-16 & $500,300,150,100$ & 2.25 \\
\hline 86 & PVCA669-70 & $500,200,150,75$ & 3.25 \\
\hline 87 & PVCA683-84 & 750,650 & 1 \\
\hline 88 & PVCA705-06 & $1300,750,700,350,150$ & 2.25 \\
\hline 89 & PVCA723-24 & $425,300,250,150$ & 1.25 \\
\hline 90 & PVCA985-86 & 200,75 & 0.25 \\
\hline 91 & PVCA1065-66 & 300,100 & 1.25 \\
\hline 92 & PVCA1077-78 & $700,350,300$ & 2.5 \\
\hline 93 & PVCA1083-84 & $500,200,100,75$ & 3.75 \\
\hline 94 & PVCA1149-50 & 1300,75 & 1 \\
\hline 95 & PVCA1191-92 & $1300,500,250$ & 2 \\
\hline 96 & PVCA1209-10* & $600,500,400,350,300,250$ & 3.5 \\
\hline 97 & PVCA1251-52 & $500,450,400,300$ & 1.5 \\
\hline 98 & PVCA1291-92 & 700,200 & 0.5 \\
\hline
\end{tabular}




\section{Continued}

\begin{tabular}{|c|c|c|c|}
\hline 99 & PVCA1333-34 & 400,225 & 2.5 \\
\hline 100 & PVCA1347-48 & 400,225 & 4.25 \\
\hline 101 & PVCA1353-54 & $350,300,150$ & 2.5 \\
\hline 102 & PVCA1355-56 & $500,300,250,125,75$ & 6.25 \\
\hline 103 & PVCA1383-84 & $650,300,200$ & 1 \\
\hline 104 & PVCA1385-86 & $700,500,450$ & 1 \\
\hline 105 & PVCA1403-04 & 800,750 & 0.75 \\
\hline 106 & PVCA1409-10 & $600,350,200,175,100$ & 6 \\
\hline 107 & PVCA1413-14 & 300,150 & 1.25 \\
\hline 108 & PVCA1455-56 & 700,125 & 2.75 \\
\hline 109 & PVCA1477-78 & $400,250,200,150$ & 2.75 \\
\hline 110 & PVCA1511-12 & $750,500,400,200,100$ & 4.5 \\
\hline 111 & PVCA1531-32 & 600 & 0.5 \\
\hline 112 & PVCA1533-34 & 350,150 & 0.75 \\
\hline 113 & PVCA1541-42 & $600,500,400,200$ & 1 \\
\hline 114 & PVCA1557-58 & $600,500,400,200$ & 3.25 \\
\hline 115 & PVCA1591-92 & $600,400,300,200$ & 1.25 \\
\hline 116 & PVCA1593-94 & $500,300,150,100$ & 3.25 \\
\hline 117 & PVCA1631-32 & $400,350,100$ & 1.25 \\
\hline 118 & PVCA1667-68 & $400,250,200,50$ & 1.75 \\
\hline 119 & PVCA1685-86 & 700,100 & 1 \\
\hline 120 & PVCA1691-92 & 500,300 & 0.5 \\
\hline 121 & PVCA1705-06 ${ }^{*}$ & $700, \mathbf{3 5 0}, 150,100$ & 2.75 \\
\hline 122 & PVCA1753-54 & $700,500,150$ & 1 \\
\hline 123 & PVCA1759-60 & 200,75 & 0.75 \\
\hline 124 & PVCA1769-70 & $600,250,150,100$ & 1 \\
\hline 125 & PVCA1781-82 & 250,200 & 1.25 \\
\hline 126 & PVCA1783-84 & $600,500,250,150$ & 1.5 \\
\hline 127 & PVCA1811-12 & $300,250,125,75$ & 5.5 \\
\hline 128 & PVCA1813-14 & 400,150 & 1.5 \\
\hline 129 & PVCA1819-20 & $700,600,550,450,400,300,150$ & 5.5 \\
\hline 130 & PVCA1827-28 & $1300,500,300,150$ & 5.75 \\
\hline 131 & PVCA1835-36 & $1300,550,150,100$ & 1.75 \\
\hline 132 & PVCA1851-52 & $200,175,125$ & 2.5 \\
\hline 133 & PVCA1853-54 & $300,250,200,150,100$ & 6 \\
\hline 134 & PVCA1867-68 & $400,300,150$ & 2.75 \\
\hline 135 & PVCA1869-70* & 325,250 & 2.25 \\
\hline 136 & PVCA1881-82 & $325,250,150$ & 1.75 \\
\hline 137 & PVCA1883-84 & 350,100 & 0.5 \\
\hline 138 & PVCA1915-16 & $300,250,150$ & 2.25 \\
\hline 139 & PVCA1925-26 & $700,400,300,150,100$ & 5.5 \\
\hline 140 & PVCA1937-38 & $400,350,250,100$ & 3.5 \\
\hline 141 & PVCA1939-40 & $700,500,450,250$ & 3.25 \\
\hline 142 & PVCA1941-42 & $400,300,200,150$ & 4.75 \\
\hline 143 & PVCA1947-48 & 400,300 & 1.75 \\
\hline 144 & PVCA1955-56 & $600,500,400,350$ & 1.5 \\
\hline 145 & PVCA1957-58 & 325,300 & 1.75 \\
\hline 146 & PVCA1963-64 & 200,175 & 2 \\
\hline 147 & PVCA1997-98 & $600,400,300$ & 1 \\
\hline 148 & PVCA2003-04 & $500,450,300,250,100$ & 2.25 \\
\hline
\end{tabular}




\section{Continued}

\begin{tabular}{|c|c|c|c|}
\hline 149 & PVCA2005-06 & $300,200,100$ & 1 \\
\hline 150 & PVCA2009-10 & 300,200 & 1 \\
\hline 151 & PVCA2013-14 & $200,150,100$ & 1 \\
\hline 152 & PVCA7-8 & 350,325 & 2 \\
\hline 153 & PVCA17-18 & $300,150,100,50$ & 2.5 \\
\hline 154 & PVCA19-20 & 100,50 & 3 \\
\hline 155 & PVCA37-38 & $300,200,100,50$ & 3 \\
\hline 156 & PVCA145-46 & $700,500,300,250,100,50$ & 5 \\
\hline 157 & PVCA151-52 & 250,150 & 0.25 \\
\hline 158 & PVCA225-26 & 75,50 & 0.75 \\
\hline 159 & PVCA243-44 & $600,500,400,300,200$ & 3 \\
\hline 160 & PVCA285-86 & $600,500,150,100,50$ & 2 \\
\hline 161 & PVCA309-10 & $750,700,550,500,200,100$ & 2.75 \\
\hline 162 & PVCA341-42 & $250,200,100$ & 3.25 \\
\hline 163 & PVCA347-48 & 350,300 & 1 \\
\hline 164 & PVCA349-50 & $700,500,150,100$ & 3.25 \\
\hline 165 & PVCA405-06 & $700,650,600,500,450,300,125$ & 6.5 \\
\hline 166 & PVCA411-12 & $650,600,300,100,50$ & 5.5 \\
\hline 167 & PVCA415-16 & 200,150 & 0.25 \\
\hline 168 & PVCA425-26 & $150,100,75$ & 2.25 \\
\hline 169 & PVCA765-66 & $550,450,400,100$ & 2 \\
\hline 170 & PVCA797-98 & 150,100 & 2 \\
\hline 171 & PVCA863-64 & $350,300,200,150,100$ & 5.25 \\
\hline 172 & PVCA931-32 & 150,75 & 2 \\
\hline 173 & PVCA1037-38 & 250,225 & 2 \\
\hline 174 & PVCA1045-46 & $175,150,50$ & 2.5 \\
\hline 175 & PVCA1187-88 & $650,300,200,150,100$ & 2.5 \\
\hline 176 & PVCA1197-98 & 450,350 & 1.7 \\
\hline 177 & PVCA1271-72 & 250,200 & 2 \\
\hline 178 & PVCA1337-38 & 350,250 & 2.25 \\
\hline 179 & PVCA1387-88 & $300,225,75$ & 3.5 \\
\hline 180 & PVCA1401-02 & 275,225 & 2 \\
\hline 181 & PVCA1405-06 & $275,250,200$ & 0.5 \\
\hline 182 & PVCA1485-86 & 200 & 1.25 \\
\hline 183 & PVCA1549-50 & 325,300 & 2 \\
\hline 184 & PVCA1589-90 & $500,225,150,75$ & 2.25 \\
\hline 185 & PVCA1605-06 & 225,200 & 1.5 \\
\hline 186 & PVCA1627-28 & 225,200 & 2 \\
\hline 187 & PVCA1669-70 & 350,300 & 1.5 \\
\hline 188 & PVCA677-78 & 325,300 & 2 \\
\hline 189 & PVCA1741-42 & 200,175 & 2 \\
\hline 190 & PVCA1883-84 & 250,175 & 2.25 \\
\hline 191 & PVCA2059-60 & $300,225,100$ & 1.5 \\
\hline 192 & PVCA2123-24 & $200,175,150$ & 3.75 \\
\hline 193 & PVCA2143-44 & 325,300 & 1.5 \\
\hline 194 & PVCA2197-98 & $225,200,175,150$ & 4.5 \\
\hline 195 & PVCA2199-2200 & 300,275 & 2 \\
\hline 196 & PVCA2207-08 & $550,500,325,200$ & 3.75 \\
\hline 197 & PVCA2239-40 & 200,175 & 2 \\
\hline 198 & PVCA2269-070 & 200,225 & 2 \\
\hline
\end{tabular}




\section{Continued}

\begin{tabular}{|c|c|c|c|}
\hline 199 & PVCA2279-80 & 200,175 & 2 \\
\hline 200 & PVCA2361-62 & 250 & 1 \\
\hline 201 & PVCA2393-94 & 200,175 & 2 \\
\hline 202 & PVCA2437-38 & 150,125 & 2 \\
\hline 203 & PVCA2461-62 & 250,200 & 2 \\
\hline 204 & PVCA2471-72 & 200, 175 & 2 \\
\hline 205 & PVCA2473-74 & 150,100 & 1.75 \\
\hline 206 & PVCA2503-04 & 250,200 & 2 \\
\hline 207 & PVCA2527-28 & 200,175 & 2 \\
\hline 208 & PVCA2615-16 & 350,325 & 2 \\
\hline 209 & PVCA2623-24* & 700,150 & 1.75 \\
\hline 210 & PVCA2647-48 & $400,250,200$ & 3.25 \\
\hline 211 & PVCA2721-22 & 250,225 & 2 \\
\hline 212 & PVCA2847-48 & $600,500,300,200$ & 1.25 \\
\hline 213 & PVCA2979-80 & $200,150,100$ & 1.25 \\
\hline 214 & PVCA3119-20 & $500,400,200$ & 3.25 \\
\hline 215 & PVCA3205-06 & 250,225 & 2 \\
\hline 216 & PVCA3327-28 & 300,250 & 2.5 \\
\hline 217 & PVCA3053-54 & 200 & 0.5 \\
\hline 218 & PVCA23-24 & 225, 200 & 0.75 \\
\hline 219 & PVCA247-48 & 150 & 1.75 \\
\hline 220 & PVCA249-50 & $300,250,200,150$ & 1 \\
\hline 221 & PVCA251-52 & 200 & 0.75 \\
\hline 222 & PVCA253-54 & $550,500,450,150$ & 3.25 \\
\hline 223 & PVCA259-60 & $750,700,600,400,250,150,100$ & 4 \\
\hline 224 & PVCA261-62 & 300,200 & 2.25 \\
\hline 225 & PVCA271-72 & 175,100 & 0.5 \\
\hline 226 & PVCA275-76 & 650, 550, 500, 450, 400, 350, 200 & 3.5 \\
\hline 227 & PVCA281-82* & $700,350,300,150$ & 1 \\
\hline 228 & PVCA283-84 & $1300,600,400,350,250,200,100$ & 6 \\
\hline 229 & PVCA287-88 & $900,800,650,400,350,150$ & 4.75 \\
\hline 230 & PVCA297-98 & $600,400,300,200$ & 3 \\
\hline 231 & PVCA299-300 & $750,700,500,450,400,150$ & 4.25 \\
\hline 232 & PVCA301-302* & $800,650,250$ & 1.25 \\
\hline 233 & PVCA307-08 & $900,800,650,450,400,350,300,150$ & 3.5 \\
\hline 234 & PVCA337-38 & 150 & 0.75 \\
\hline 235 & PVCA343-44 & $600,300,200$ & 2 \\
\hline 236 & PVCA345-46 & 450,200 & 0.5 \\
\hline 237 & PVCA351-52 & $900,650,150$ & 0.75 \\
\hline 238 & PVCA357-58 & $700,550,150$ & 1 \\
\hline 239 & NFSG-105 & 200, 175 & 3 \\
\hline 240 & NFSG-26 & 150,125 & 3 \\
\hline 241 & SWW-177 & 200,175 & 2 \\
\hline 242 & SWW-108 & 175,150 & 0.5 \\
\hline 243 & NFSG-50 & 175,150 & 0.5 \\
\hline 244 & NFSG-125 & 175,150 & 2 \\
\hline 245 & SWW-2945 & $600,350,300,250$ & 1.5 \\
\hline 246 & SWW-2906 & 275,50 & 1 \\
\hline 247 & NFSG-36 & $500,225,175$ & 1.5 \\
\hline 248 & NFSG-54 & 600,150 & 2 \\
\hline
\end{tabular}




\begin{tabular}{clcc} 
Continued & & & \\
\hline 249 & NFSG-288 & 175,150 & 2 \\
250 & NFSG-246 & 100,50 & 0.25 \\
251 & NFSG-145 & 250,225 & 2 \\
252 & SWW-1394 & 250,200 & 2.25 \\
253 & NFSG-133 & 175,150 & 0.75 \\
254 & NFSG-132 & 325,300 & 1.75 \\
\hline
\end{tabular}

\#Data published by Wang et al. 2011 [25] and Okada et al.2010 [24]. *Amplified allele (marked bold) size in proso millet = allele size in switchgrass [25].

conserved and syntenic between switchgrass and proso millet, which suggests amplification of either orthologous or paralogus genes [31].

Most of the polymorphic markers (181 out of 254) produced 2, 3 or 4 alleles and 73 markers have produced 1, 5, 6, 7 or 8 alleles. Total numbers of alleles amplified by each SSR marker on the set of 8 lines ranged from 1 to16. Highest alleles (16) were amplified by marker PVCA 1221-22. High frequency of SSR markers with 2 alleles also indicates diploid nature of proso millet genome. These indicate that proso millet genome is not $100 \%$ tetraploid but rather partially tetraploid and partially diploid although proso millet was reported as allotetraploid [32]. Amplification of multiple loci by a few SSR markers might be due to the multiple primer binding sites throughout the genome or within a single locus, which may be due to gene duplication or duplicated genomic regions [17]. This is in agreement with previous reports on proso millet, potato, and zoyagrass species [15, 27,33].

The 254 polymorphic SSR markers amplified a total of 984 alleles (amplified DNA markers) among the set of 8 proso millet genotypes. Sizes of alleles by the switchgrass SSRs in proso millet ranged from $50 \mathrm{bp}$ to $1300 \mathrm{bp}$ with varied number of bands of each size. Amplified DNA bands in ranges of 50 - 200 bp, 200 - 400 bp, 400 600 bp, $600-800$ bp, $800-1000$ bp, >1000 bp were 377 (38\%), 325 (33\%), 138 (14\%), 98(10\%), 23 (2\%) and 23(2\%), respectively. When SSR marker sizes in proso millet were compared with expected switchgrass sizes, only 9 markers (PVCA1535-36PVCA1565-66,

PVCA1731-32, PVCA1209-10, PVCA1705-06, PVCA1869-70, PVCA2623-24, PVCA281-82,

PVCA301-302) showed exact matches with that of switchgrass and majority of marker sizes were different in proso millet. This high level of differences indicates significant genome reorganization in proso millet after speciation during evolution which was reported in wheat [34].

\subsection{SSRs and Possible Association with Abiotic Stresses}

We found that eight SSR markers are part of the mRNA, UniGenes or genomic sequences in other crops (Table 2).
The SSR marker PVCA1017-1018 has 100\% sequence similarity with the mRNA sequence involved in glutathione metabolism in sorghum. Another SSR marker PVCA723-724 is located on the rice BAC clone from where $S u b-1$ gene was cloned. Glutathione metabolism in sorghum and Sub-1 gene in rice were reported to be associated with drought and flood tolerance, respectively $[35,36]$. It is likely that, these genes are still present in the proso millet genome and are showing different mechanism for drought tolerance. This evolutionary conservation for these genes provides a valuable source for further marker development for abiotic stresses in proso millet.

\subsection{Repetitive DNA in Proso Millet}

Repeat motifs of the 254 polymorphic SSR primers in proso millet were analyzed to elucidate the type of repetitive DNA in proso millet. Of the total 254 polymorphic markers 221 (87\%) were di-nucleotide repeats and the remaining 33 (13\%) were tri- and other composite repeats (Table 3). Of all these repeat-motifs, 196 were perfect $(77 \%)$ and 58 were compound $(23 \%)$ repeats. Of the 221 di-nucleotide motifs, the most abundant repeatmotif was AG/GA (47\%) followed by AC/CA (22\%) repeat motif in proso millet. Together the AG/GA and AC/CA repeat motif contributed $69 \%$ of all the polymorphic markers in proso millet. The third most abundant di-nucleotide repeats in proso millet were GT/TG and CT/TC repeats, which together was $11 \%$. All the remaining di-nucleotide repeats accounted for $7 \%$ of all the repeats.

A high level of di-nucleotide repeats in polymorphic SSRs in proso millet matches with other reports where $(\mathrm{CA} / \mathrm{GT})_{\mathrm{n}}$ and $(\mathrm{CT} / \mathrm{AG})_{\mathrm{n}}$ were predominant repeat motifs and were found in the euchromatin and hetrochromatin regions, respectively [37]. The AG/GA repeats were shown to be related to drought tolerance in rice [38]. Therefore, it may not be surprising that AG/GA repeats are the pre-dominant repeat motifs in proso millet since proso millet is known to have good tolerance to low soil moisture condition. This result is similar to the report presented by Cho et al. and 42\% AC/CA repeats. However, it is opposed to AT/TA and CT/GA repeats as predominant repeats in other plants $[39,40]$. 
Table 2. Eight switchgrass SSR markers, which are polymorphic in proso millet and their possible functional association in other crops based on sequence similarities available in NCBI data base.

\begin{tabular}{ccccc}
\hline SSR markers & Crop & Accession No. & $\begin{array}{c}\text { Sequence type } \\
\text { (gDNA/mRNA) }\end{array}$ & $\begin{array}{c}\text { Putative protein } \\
\text { /gene/location }\end{array}$ \\
\hline PVCA275-276 & Sorghum & XM_002466569.1 & mRNA & Hypothetical protein \\
PVCA275-276 & Oat & JN390967.1 & mRNA & Moxygenase \\
PVCA429-430 & Wheat & GU985444.1 & gDNA & Uncharacterized \\
PVCA495-496 & Maize & BT065995.2 & mRNA & Sub1 locus \\
PVCA723-724 & Rice & DQ453964.1 & gDNA & mRNA \\
PVCA1017-1018 & Sorghum & XM_002465397.1 & mRNA & $\begin{array}{c}\text { Hypothetical protein (predicted to be } \\
\text { involved in glutathione metabolism) }\end{array}$ \\
PVGA1119-1120 & Barley & AK373276.1 & Predicted protein \\
PVCA579-580 & Arabidopsis & CP002687.1 & & $\begin{array}{c}\text { Peroxidase 38 Tetratricopeptide } \\
\text { repeat domain-containing protein } \\
\text { Myosin heavy chain-like protein }\end{array}$ \\
\hline
\end{tabular}

Table 3. 254 polymorphic SSR markers and their type and repeat-motifs, and its relative frequency in proso millet.

\begin{tabular}{|c|c|c|c|}
\hline Repeat unit & Repeat type $^{\#}$ & Number of markers & Frequency (\%) \\
\hline \multirow[t]{13}{*}{ Di-Nucleotide (221) } & AG or GA & 120 & 47.24 \\
\hline & $\mathrm{AC}$ or $\mathrm{CA}$ & 57 & 22.44 \\
\hline & GT or TG & 18 & 7.09 \\
\hline & CT or TC & 10 & 3.94 \\
\hline & AC/AG, CA/GA, CA/AG, GA/CA & 7 & 2.76 \\
\hline & $\mathrm{AC} / \mathrm{AT}$ & 2 & 0.79 \\
\hline & AG/GT, GT/GA & 2 & 0.79 \\
\hline & CT/AG & 1 & 0.39 \\
\hline & CT/GT & 1 & 0.39 \\
\hline & GT/AC & 1 & 0.39 \\
\hline & $\mathrm{TA} / \mathrm{AT}$ & 1 & 0.39 \\
\hline & TA/TG & 1 & 0.39 \\
\hline & CT/AC & 1 & 0.39 \\
\hline Sub-total & & 221 & $87 \%$ \\
\hline \multirow[t]{11}{*}{ Tri-Nucleotide (30) } & GAA/AAG & 3 & 1.18 \\
\hline & AGC,CAG,GCA, ACA & 5 & 1.97 \\
\hline & TGC,GCT,CTG & 6 & 2.36 \\
\hline & ATC,CAT & 2 & 0.79 \\
\hline & CCG,CGC & 2 & 0.79 \\
\hline & TCT & 4 & 1.57 \\
\hline & GGT & 1 & 0.39 \\
\hline & $(\mathrm{TGC})_{5}-(\mathrm{CTG})_{5},(\mathrm{TGC})_{9}-(\mathrm{TGC})_{6}$ & 2 & 0.79 \\
\hline & $(\mathrm{TCC})_{5}-(\mathrm{TCC})_{5},(\mathrm{TCC})_{7}-(\mathrm{TGC})_{8}$ & 2 & 0.79 \\
\hline & $(\mathrm{GCT})_{8}-(\mathrm{CTG})_{5}$ & 1 & 0.39 \\
\hline & $(\mathrm{CAG})_{5}-(\mathrm{GCA})_{6}$ & 1 & 0.39 \\
\hline Others (tetra,penta, hexa-nucleotide) (3) & & 3 & 1.18 \\
\hline Sub-total & & 33 & $13 \%$ \\
\hline Total & & 254 & $100 \%$ \\
\hline
\end{tabular}

${ }^{\#}$ Repeat motifs from Wang et al. 2011 [25] and Okada et al. 2010 [24]. 


\section{Conclusion}

To the best of our knowledge, this is the first report of such a large set (339) of SSR markers for proso millet using cross species genomic resources. This will help to advance proso millet genomic research and will ultimately lead to genetic improvement of this crop.

\section{Acknowledgements}

This research was funded by the Nebraska Wheat Board and Cross Road Coop and Friends of Proso Millet Research Fund. Partial funding for D.K. Santra was from Hatch project (NEB-43-105) and the graduate research assistancefor S.G. Rajput was from the Agronomy and Horticulture Department of the University of Nebraska-Lincoln.

\section{REFERENCES}

[1] A. Lawler, "Bridging East and West: Millet on the Move," Science, Vol. 325, No. 5943, 2009, pp. 942-943. http://dx.doi.org/10.1126/science.325_942

[2] G. W. Wiegrefe, "How to Prduceproso Millet (A Farmer's Guide),” Pierre, South Dakota, 1990.

[3] D. D. Baltensperger, "Progress with Proso, Pearl and Other Millets,” In: J. Janick and A. Whipkey, Eds., Trends in New Crops and New Uses, ASHS Press, Alexandria, 2002, pp. 100-103.

[4] E. A. Oelke, E. S. Oplinger, D. H. Putnam, B. R. Durgan, J. D. Doll and D. J. Undersander Millets, “Alternative Field Crops Manual,” University of Wisconsin, Cooperative Extension and University of Minnesota, Center for Alternative Plants \& Animal Products and Minnesota Extension Service, 1990.

[5] M. Brink and G. Belay, "Cereals and Pulses,” Plant Resources of Tropical Africa, 2006, pp. 122-125.

[6] D. J. Lyon, P. A. Burgener, K. L. De Boer, R. M. Harveson, G. L. Hein, G. W. Hergert, T. L. Holman, L. A. Nelson, J. J. Johnson, T. Nleya, J. M. Krall, D. C. Nielsen and M. F. Vigil, "Producing and Marketing of Proso Millet in Great Plains,” 2008. http://ianrpubs.unl.edu/live/ec137/build/ec137.pdf

[7] USDA-NASS Quick Stats. http://quickstats.nass.usda.gov/results/962608D8-F138-3 CC7-9705-068D2A399631

[8] D. K. Santra and D. Rose, "Alternative Uses of Proso Millet,” University of Nebraska-Lincoln NebGude G2218, 2013.

[9] AGMRC, 2012. http://www.agmrc.org/commodities_products/grains_oi lseeds/proso-millet/

[10] H. K. M. Ribu and K. W. Hilu, "Detection of Interspecific and Intraspecific Variation in Panicummillets through Random Amplified Polymorphic DNA,” Theoretical and Applied Genetics, Vol. 88, 1994, pp. 412-416.

[11] D. Karam, P. Westra, S. J. Nissen, S. M. Ward and J. E. F.
Figueiredo, "Genetic Diversity Among Proso Millet (Panicum miliaceum L.) Biotypes Assessed by AFLP Technique,” Planta Daninha, Vol. 22, No. 2, 2004, pp. 167174. http://dx.doi.org/10.1590/S0100-83582004000200001

[12] D. Karam, P. Westra, S. J. Niessen, M. W. Sarah and J. E. F. Figueiredo, “Assessment of Silver-Stained AFLP Markers for Studying DNA Polymorphism in Proso Millet (Panicum miliaceum L.)," Revista Brasileira de Botanica, Vol. 29, No. 4, 2006, pp. 609-615.

[13] W. Powell, M. Morgante and C. Andre, "The Comparison of RFLP, RAPD, AFLP and SSR (Microsatellite) Markers for Germplasm analysis,” Molecular Breeding, Vol. 2, No. 3, 1996, pp. 225-238. http://dx.doi.org/10.1007/BF00564200

[14] P. K. Gupta and R. K. Varshney, "The Development and Use of Microsatellite Markers Fore Genetic Analysis and Plant Breeding with Emphasis on Bread Wheat,” Euphytica, Vol. 113, No. 3, 2000, pp. 163-185. http://dx.doi.org/10.1023/A:1003910819967

[15] X. Hu, J. Wang, P. Lu and H. Zhang, “Assessment of Genetic Diversity in Broomcorn Millet Panicum miliaceum L.) Using SSR Markers,” Journal of Genetics and Genomics, Vol. 36, No. 8, 2009, pp. 491-500. http://dx.doi.org/10.1016/S1673-8527(08)60139-3

[16] C. Young-II, J.-W. Chung, G.-A. Lee, K.-H. Ma, A. Dixit, J.-G. Gwag and Y.-J. Park, "Development and Characterization of Twenty-Five New Polymorphic Microsatellite Markers in Proso Millet (Panicum miliaceum L.)," Genes \& Genomics, Vol. 32, No. 3, 2010, pp. 267-273. http://dx.doi.org/10.1007/s13258-010-0007-8

[17] M. C. Saha, J. D. Cooper, M. A. RoufMian, K. Chekhovskiy and G. D. May, "Tall Fescue Genomic SSR Markers: Development and Transferability across Multiple Grass Species," Theoretical and Applied Genetics, Vol. 113, No. 8, 2006, pp. 1449-1458. http://dx.doi.org/10.1007/s00122-006-0391-2

[18] L. Zane, L. Bargelloni and T. Patarnello, "Strategies for Microsatellite Isolation: A Review," Molecular Ecology, Vol. 11, No. 1, 2002, pp. 1-16. http://dx.doi.org/10.1046/j.0962-1083.2001.01418.x

[19] S. H. Dwivedi, H. Upadhyaya, S. Senthilvel, C. Hash, K. Fukunaga, X. Diao, D. K. Santra, D. Baltensperger and M. Prasad, "Millets: Genetic and Genomic Resources," Plant Breeding Review, Vol. 6, 2012, pp. 247-375.

[20] S. L. Dillon, P. K. Lawrence and R. J. Henry, “The New Use of Sorghum bicolor-Derived SSR Markers to Evaluate Genetic Diversity in 17 Australian Sorghum Species,” Plant Genetic Resources: Characterization and Utilization, Vol. 3, No. 1, 2005, pp. 19-28. http://dx.doi.org/10.1079/PGR200454

[21] C. C. Tan, Y. Q. Wu, T. Charles, A. Michael, T. Chuck and T. Samuels, "Development of Simple Sequence Repeat Markers for Bermuda Grass from Its Expressed Sequence Tag Sequences and Preexisting Sorghum SSR Markers," Molecular Breeding, Vol. 29, No. 1, 2012, pp. 23-30. http://dx.doi.org/10.1007/s11032-010-9521-2

[22] E. C. Bess, A. N. Doust and E. A. Kellogg, “A Naked Grass in the 'Bristle Clade': A Phylogenetic and Deve- 
lopmental Study of Panicum Section Bulbosa (Paniceae: Poaceae)," International Journal of Plant Sciences, Vol. 166, No. 3, 2005, pp. 371-381. http://dx.doi.org/10.1086/428701

[23] L. Liu, Y. Wu, Y. Wang and T. Samuels, “A High-Density Simple Sequence Repeat-Based Genetic Linkage Map of Switchgrass,” Genes Genomes and Gentics, Vol. 2, No. 3, 2012, pp. 357-370.

[24] M. Okada, C. Lanzatella, M. C. Saha, J. Bouton, R. Wu and C. M. Tobias, "Complete Switchgrass Genetic Maps Reveal Subgenome Collinearity, Preferential Pairing and Multilocus Interactions,” Genetics, Vol. 185, No. 3, 2010, pp. 745-760. http://dx.doi.org/10.1534/genetics.110.113910

[25] Y. W. Wang, T. D. Samuels and Y. Q. Wu, "Development of 1,030 Genomic SSR Markers in Switchgrass," Theoretical and Applied Genetics, Vol. 122, No. 4, 2011, pp. 677-686. http://dx.doi.org/10.1007/s00122-010-1477-4

[26] J. J. Doyle and J. L. Doyle, "A Rapid DNA Isolation Procedure from Small Amount of Fresh Leaf Tissue," Phytochemical Bulletin, Vol. 19, 1987, pp. 11-15.

[27] A. Prevost and M. J. Wilkinson, “A New System of Comparing PCR Primers Applied to ISSR Fingerprinting of Potato Cultivars,” Theoretical and Applied Genetics, Vol. 98, No. 1, 1999, pp. 107-112.

http://dx.doi.org/10.1007/s001220051046

[28] J. Yu, M. La Rota, R. Kantety and M. Sorrells, "EST Derived SSR Markers for Comparative Mapping in Wheat and Rice," Molecular Genetics and Genomics, Vol. 271, No. 6, 2004, pp. 742-751. http://dx.doi.org/10.1007/s00438-004-1027-3

[29] A. L. S. Azevedo, P. P. Costa, J. C. Machado, M. A. Machado, A. V. Pereira and F. J. da Silva Lédo, "Cross Species Amplification of Pennisetumglaucum Microsatellite Markers in Pennisetumpurpureum and Genetic Diversity of Napier Grass Accessions," Crop Science, Vol. 52, No. 4, 2012, pp. 1776-1785. http://dx.doi.org/10.2135/cropsci2011.09.0480

[30] M. L. Wang, N. A. Barkley, J.-K. Yu, R. E. Dean, M. L. Newman, M. E. Sorrells and G. A. Pederson, "Transfer of Simple Sequence Repeat (SSR) Markers from Major Cereal Crops to Minor Grass Species for Germplasm Characterization and Evaluation," Plant Genetic Resources: Characterization and Utilization, Vol. 3, No. 1, 2005, pp. 45-57. http://dx.doi.org/10.1079/PGR200461

[31] R. K. Varshney, A. Graner and M. E. Sorrells, “Genic Microsatellite Markers in Plants: Features and Applications," Trends in Biotechnology, Vol. 23, No. 1, 2005, pp.
48-55. http://dx.doi.org/10.1016/j.tibtech.2004.11.005

[32] H. V. Hunt, H. M. Moots, R. A. Graybosch, H. Jones, M. Parker, O. Romanova, M. K. Jones, C. J. Howe and K. Trafford, "Waxy Phenotype Evolution in the Allotetraploid Cereal Broomcorn Millet: Mutations at the GBSSI Locus in Their Functional and Phylogenetic Context," Molecular Biology and Evolution, Vol. 30, No. 1, 2013, pp. 109-122. http://dx.doi.org/10.1093/molbev/mss209

[33] H. W. Cai, M. Inoue, N. Yuyama, W. Takahashi, M. Hirata and T. Sasaki, "Isolation, Characterization and Mapping of Simple Sequence Repeat Markers in Zoysiagrass (Zoysiaspp.)," Theoretical and Applied Genetics, Vol. 112, No. 1, 2005, pp. 158-166. http://dx.doi.org/10.1007/s00122-005-0118-9

[34] M. Feldman, B. Liu, G. S. Segal, A. Abbo, A. Levy and J. M. Vega, "Rapid Elimination of Low-Copy DNA Sequences in Polyploid Wheat: A Possible Mechanism for Differentiation of Homoeologous Chromosomes," Genetics, Vol. 147, No. 3, 1997, pp. 1381-1387.

[35] Y. Chi, Y. Cheng, J. Vanitha, N. Kumar, R. Ramamoorthy, S. Ramachandran and S.-Y. Jiang, "Expansion Mechanisms and Functional Divergence of the Glutathione S-Transferase Family in Sorghum and Other Higher Plants," DNA Research, Vol. 18, No. 1, 2011, pp. 1-16. http://dx.doi.org/10.1093/dnares/dsq031

[36] T. Fukao, E. Yeung and J. Bailey-Serres, “The Submergence Tolerance Regulator SUB1A Mediates Crosstalk between Submergence and Drought Tolerance in Rice," Plant Cell, Vol. 23, No. 1, 2011, pp. 412-427. http://dx.doi.org/10.1105/tpc.110.080325

[37] A. Cuadrado and N. Jouve, "Similarities in the Chromosomal Distribution of AG and AC Repeats within and between Drosophila, Human and Barley Chromosomes," Cytogenetic and Genome Research, Vol. 119, No. 1-2, 2007, pp. 91-99. http://dx.doi.org/10.1159/000109624

[38] C. S. Reddy, B. A. Prasad, B. P. M. Swamy, K. Kaladhar and N. Sarla, "ISSR Markers Based on GA and AG Repeats Reveal Genetic Relationship among Rice Varieties Tolerant to Drought, Flood or Salinity," Journal of Zhejiang University Science, Vol. 2, 2009, pp. 133-141.

[39] L. Cardle, L. Ramsay, D. Milbourne, M. Macaulay, D. Marshall and R. Waugh, "Computational and Experimental Characterization of Physically Clustered Simple Sequence Repeats in Plants,” Genetics, Vol. 156, 2000, pp. 847-854.

[40] P. Y. Danin, N. Reis, G. Tzuri and N. Katzir, "Devolopment and Characterization of Microsatellites Markers in Cucumis," Theoretical and Applied Genetics, Vol. 102, 2001, pp. 61-72. 